\title{
The Possible Benefits of Resistance Training in Counteracting the Age-Related Biological Decline of Skeletal Muscle
}

\author{
Reyes M. Beltran Valls and Daniela Caporossi
}

As a result of the increased human lifespan expectancy, age-related disabilities and the associated medical care costs represent in many countries a major economic concern because of the increased national health expenditures utilized by the elderly. In normal aging, the phenomenon of sarcopenia, associated to loss of skeletal muscle mass and strength with a reduction in motor unit number and atrophy of muscle fibres, results in the decline of physical function and quality of life. Regular physical activity has been shown to have beneficial effects against the development of many age-related chronic diseases in men and women and it seems to be of great value also for the skeletal muscle mass, however we need to clearly understand the mechanisms affecting muscle wasting and how programmed exercise can delay or counteract this process. This mini-review aims to offer an overview on the biological mechanisms underlying the age-associated loss of muscle mass and physical function and to examine how resistance exercise could be a potential kinesiology intervention for the elderly.

\section{Introduction}

A ging is a complex process among which several factors may interact simultaneously and may operate at different functional levels. From a biological point of view, aging is a process that results from a reduced capacity to maintain the cellular homeostasis in presence of adverse stress, thus generating impairments related to risk factors and diseases. The study on aging has been expanded due to the increased human lifespan average. As a result, age-related disabilities and the associated medical care costs are a major economic concern because of the increased national health expenditures utilized by the elderly.

According to the United Nations, "Population ageing is unprecedented, without parallel in the history of humanity...". By 2050, the number of older people in the world will exceed the number of young for the first time in history".

Daniela Caporossi, Professor with the Department of Health and Sciences, University of Rome "Foro Italico", Rome, Italy, Reyes M. Beltran Valls is also associated with the Department of Health and Sciences, University of Rome "Foro Italico", Rome, Italy
This shift towards increased life expectancy associated with a sedentary lifestyle may affect societies, economies, lives of patients and the practice of medicine. Prolongation of life expectancy is a fundamental aim for the public health care system; however it would be also relevant to consider the importance of maintaining independent living and well functioning when extending life.

The skeletal muscle corresponds to almost half of the human body mass and since it has a central role for human mobility and is a major site of metabolic activity, deterioration of the contractile material and the metabolic function have relevant consequences on human health and elderly independence. Loss of muscle mass is commonly found in people after 60 years old, and it is related to declines in physical function and quality of life. The skeletal muscle is directly affected by the age-related biological changes that affect organs and systems supporting its functionality, such as neural, endocrine and cardiovascular systems, and it is as well affected by the reduction of physical activity (Bonetto et al., 2009). Sarcopenia, the loss in muscle mass and strength associated with aging (Rosenberg, 1997), reflects a progressive 
decrease of anabolism and an increased catabolism rate, together with a reduced regeneration capacity of the tissue (Narici \& Maffulli, 2010). Due to the detrimental effects of sarcopenia, it is of outmost importance to identify the most effective means to postpone the deleterious effects of reduced muscle mass and physical frailty in old age. Frailty is a multidimensional syndrome that involves decreased reserve capacity and resistance to stressors, resulting from age-related cumulative decline across multiple physiologic systems and impaired homeostatic capacity that increase vulnerability to adverse outcomes like falls, hospitalization, institutionalization and mortality (Bauer \& Sieber 2008; Fried et al. 2001). Regular physical activity has been shown to protect against diverse components of the frailty syndrome in men and women of all ages (Peterson et al. 2009). Frailty is not a contraindication to physical activity; rather physical exercise prescription may be significantly beneficial for frail people. Having an active lifestyle seems to be of great value. Exercise generates numerous physiological adaptations that may transfer to improvements in clinical outcomes such as reduced risk of falls, improved walking speed, optimal blood analysis results, etc, however the clear cellular and molecular mechanisms supporting these benefits are not well determined yet. In addition, we need to clearly understand the mechanisms affecting muscle wasting and how programmed exercise could modify them (Kjaer \& Jespersen, 2009).
The aim of this paper is to review the biological mechanisms underlying the age-associated loss of muscle mass and physical function and to examine how resistance exercise could be a potential kinesiology intervention for the elderly to counteract this situation that may lead to a frail condition.

\section{Physiological Changes During Normal Human Aging}

Function of most physiological systems decline with aging leading to limited physical capacity. According to the World Health Organization (WHO) model, functional capacity over the course of life reaches its peak at early adulthood (20 to30-year-olds) and decreases steadily with advancing age. At a certain point of decline, it reaches the disability threshold. However, the rate of decline is highly individual and could be substantially affected both by intrinsic factors (e.g. genetic factors, physiologic changes with aging, diseases and impairments) and by the environmental situation (e.g. social, behavioural and economic factors) (WHO, 2001). Maintenance of independent living, specially delaying or preventing frailty, needs clear target interventions that slow down the age-associated decline of functional capacity to prolong the disability-free lifespan. An example is shown in the Figure 1 with respect to skeletal muscle.

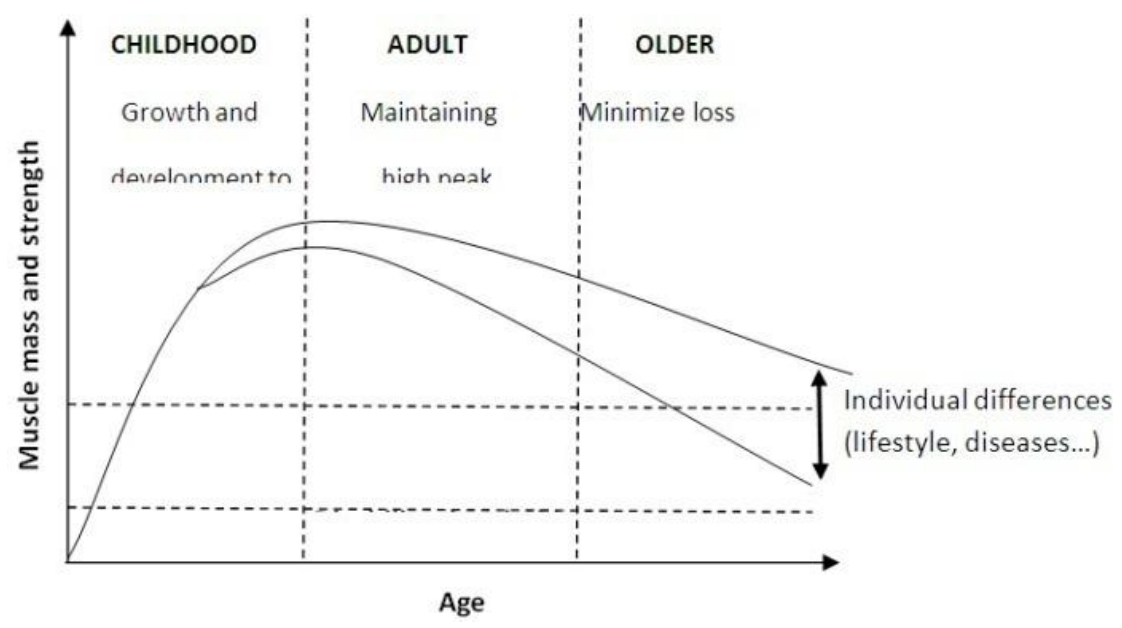

Figure 1 - Muscle mass and strength increase gradually until the adult age when peak capacity is reached. The higher the peak the higher the threshold point from where decline starts. (Modified from $W H O, 2001$ ) 
The aim of maintaining muscle mass and strength along the adult life would be to reach the disability threshold as late as possible to ensure quality of life. Physical activity, and concretely resistance exercise appears to slow down the decline of muscle-skeletal impairments in the older life (Johnston et al., 2008).

The age-related modifications of the cardiovascular and muscular system, as well as the somatosensory system, have been very much studied due to their implication in daily life activities. Regarding the cardiovascular system, there is a dramatic decline in maximal aerobic capacity $\left(\mathrm{VO}_{2 \max }\right)$ with aging, approximately $10 \%$ per decade after the third decade, due to decreased cardiac output, reduced maximal heart rate and ejection fraction as well as loss of skeletal muscle mass and muscle oxygen uptake (Singh, 2004). Unfortunately, the molecular basis for these reductions are unknown (Booth \& Zwetsloot, 2010). In addition, the decline in aerobic capacity occurring with the aging process is maximized with physical inactivity. The physical demand for a determined task is unaltered with time however, while maximal oxygen uptake is reduced due to the aging process, and thus low physical demanding tasks require progressively higher percentage of the maximal oxygen consumption. As a result the metabolic cost for a given motor task increases for an elderly person and the ability to carry out motor daily tasks is concerned since they might be performed almost at maximal percentage of the oxygen consumption (Puggaard, 2005). The combined decrease of $\mathrm{VO}_{2 \max }$, muscular strength and power contribute to the loss of function during aging.

On the other hand, the sensory systems, in combination to strength and reaction time capacities, are significant components for balance control, which is deeply affected by the process of aging. Instability in older people can result from impaired sensory, motor and central processing systems (Sturnieks et al., 2008). A part from the later on mentioned changes affecting the strength and power generation capacities as well as the neural changes affecting information processing, age-related sensory deficiencies that disturb vision, vestibular sense and proprioception may concern balance, gate and posture maintenance.

There is also wide interest in understanding agerelated changes in skeletal muscle quantity and quality. Age-associated processes affecting the muscle tissue alter the mass, the composition, the contractile properties as well as the tendons structure. The loss of muscle mass is a consequence of atrophy of the individual muscle fibers and loss of muscle fiber due to denervation or apoptosis, with a preferential atrophy of type 2 fast twitch fibers (Burton \& Sumukadas, 2010). Denervation of motor units which are renervated with slow motor units can lead to increased muscle fatigability. These changes translate into alterations of muscle power, strength and physical function and lead to reduced performance of daily living activities, decreased physical performance, increased risk of fall-related injuries and frailty (Lang et al., 2010; Boire, 2009). Deterioration of activities such as rising from a chair, climbing steps or regaining posture after perturbation of balance increase the risk of falling and the loss of independence. In addition, metabolic deficits result in decreased metabolic rate and oxygen consumption augmenting the risk of insulin resistance, type 2 diabetes and obesity (Kjaer \& Jespersen, 2009). Luckily, research shows that some of these changes, but not all, may be neutralized with higher levels of physical exercise, especially with resistance training (Phillips, 2007).

There is now much evidence to support the hypothesis that behind these muscular changes there is a multi-factorial pathogenesis, but more information is needed about the individual contribution of each biological process. A complex interplay of factors such as endocrine changes, oxidative stress and chronic inflammation as well as exogenous factors like inactivity and malnutrition may alter the physiological balance between protein synthesis and degradation (Bonetto et al., 2009).

\section{Age-Related Cellular and Molecular Changes of the Skeletal Muscle}


Although the specific contribution of each of the mentioned factors is very difficult to establish, it is generally stated that loss of muscle mass at molecular level is the result of an unbalance between the rate of protein synthesis and degradation. The loss of this equilibrium favouring degradation may result in muscle mass loss (atrophy), however when a net positive balance exists and protein synthesis exceeds protein degradation the cross-sectional area of the pre-existing muscle fibres increases by accumulation of contractile proteins (hypertrophy) (Saini et al., 2009). Nevertheless, there are several complex biochemical and genetic regulators of this equilibrium controlling anabolism and catabolism of muscle mass that may be modified during the aging process (Fig.2).

Aging is associated with altered endocrine, inflammatory and other intrinsic factors that may affect maintenance and regeneration of skeletal muscle mass. Loss of muscle mass and function may be accelerated by causes such as anabolic resistance due to the decline in insulinlike growth factor I (IGF-I) which is a promoter of protein synthesis in skeletal muscle, as well as protein degradation due to chronic increased levels of inflammatory cytokines, increased oxidative damage caused by mitochondrial dysfunction and failure of muscle regenerative capacity due to changes in molecular regulators of muscle satellite cells (Lang et al., 2010). These are a combination of systemic factors influencing the properties of muscle mass and strength eventually resulting in mobility impairments and loss of physical function. Recently, disuse (resulting from a sedentary lifestyle) has been also considered as an important factor influencing atrophy and luckily this process can be reversed with higher levels of physical activity (Kjaer \& Jespersen, 2009). The effects of ageing on skeletal muscle appear to be inevitable and it is unclear whether these intrinsic changes are unalterable or reversible. Strength, power and functional capacities are dependent on maintenance or recovery of skeletal muscle mass and its characteristics. Hence, to this aim therapies should intend to attenuate muscle protein breakdown or to stimulate muscle protein anabolism.

While the complete understanding of sarcopenia needs to be elucidated yet, one potential mechanism involves the age-related decline in muscle regenerative capacity. The human skeletal muscle has a remarkable capacity to regenerate following damage or atrophy due to the presence of myogenic stem cells known as satellite cells (Charge \& Rudnicki, 2004). When satellite cells are activated, they proliferate and differentiate providing supplementary myonuclei that fuse with the damaged myotubes. When damaged myotubes are replaced, no further proliferation occurs and the satellite cells partially return to their quiescent state waiting for further damage activation. IGFI, as well as other circulating factors, are believed to facilitate this process of repair by increasing satellite cell's proliferation, survival and differentiation capacity (Saini et al., 2009). 


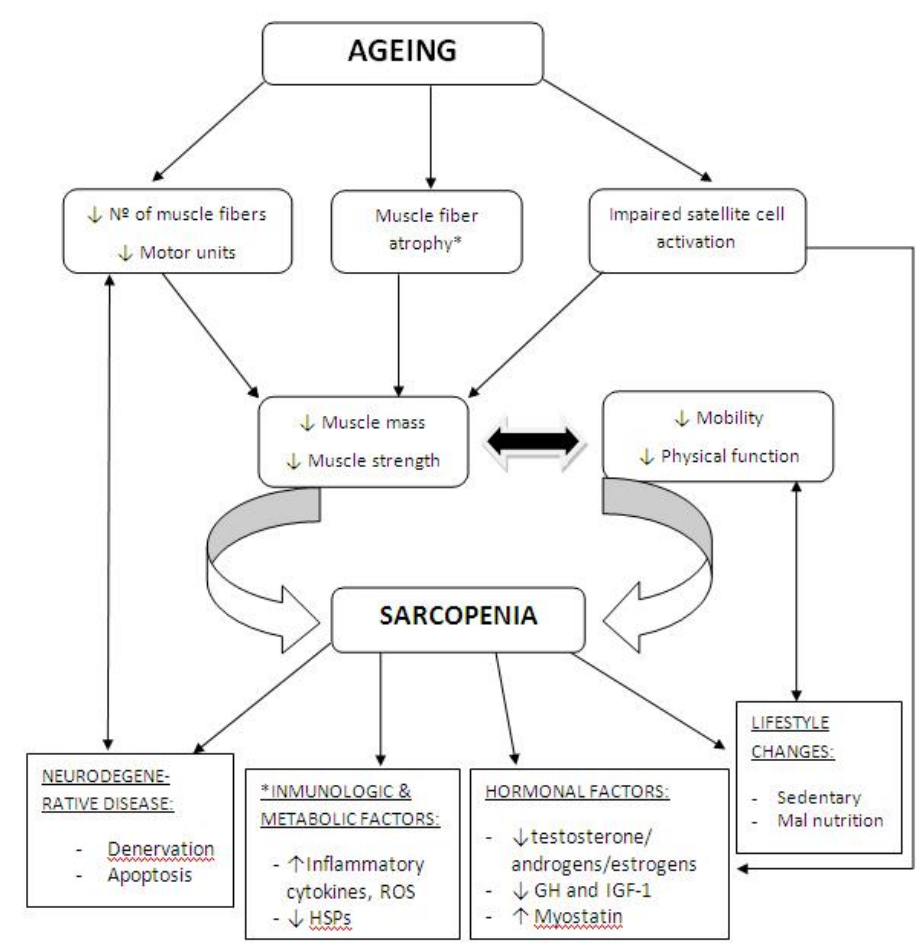

Figure 2 - Scheme summarizing the multi-factorial pathogenesis of skeletal muscle during aging (Modified from Narici \& Maffulli, 2010)

The regeneration process of skeletal muscle in aging humans is found to be faulty, what contributes to the loss of muscle mass. It has been showed recently that failure of skeletal muscle maintenance and repair in aged animals and humans may be more due to age-related changes in the molecular regulators of the differentiation process (myogenic regulator factors) than to age-related lessen in satellite cell function or potential (Conboy et al., 2005). It suggests that the capacity of muscle to regenerate is not altered by aging, but the vital environment for it does so. (Conboy et al., 2005; Carlson et al., 2009). Satellite cells activation and proliferation is mediated by Notch, a trans-membrane receptor which activation declines with aging. Notch activation is dependent on mitogen-activated protein kinase (MAPK) activity, a transduction signal that also appears to be reduced. In addition, transforming growth factor beta (TGF- $\beta$ ) shows increased levels in older individuals what causes accumulation of the enzymatic protein cyclindependent kinase, which inhibits satellite cells and hampers their regenerative response to injury (Carlson et al., 2009). Moreover, agerelated loss of muscle mass has been confirmed to be associated with replacement of connective tissue due to a reduced regeneration capacity and increased fat mass (Carlson et al., 2009).

On the other hand, hormonal factors that promote protein synthesis show decreased expression associated with senescence. Reduced levels of IGF-I may be a key factor inducing the molecular cascade of events that lead to muscle atrophy, since IGF-I stimulates intramuscular mediators of protein synthesis in the skeletal muscle and reduces protein degradation rate (Saini et al., 2009; Bonetto et al., 2009). Research suggests that low circulating IGF-1 bioactivity and abnormalities of IGF-1 signalling in elderly subjects, especially in individuals with a pro-inflammatory state, are associated with age-related muscle wasting. It may be caused by impaired activation of anabolic signalling pathways from the GH/IGF1 axis that involve, among others, the IRSs (receptors substrates), PI3-K (phosphatidylinositol 3-kinase), and AKT (antiapoptotic serine protein kinase B), (Perrini et al., 2010).

Mice lacking of GHR (growth hormone receptor) exhibit proportional growth retardation and delayed and/or diminished 
responses in IRS-1 phosphorylation following in vivo insulin stimulation in muscle (Robertson et al. 2006). Decreased levels of PI3-K in the muscle result in decreased muscle size and inefficient muscle metabolism (Luo et al., 2006) and an age-related deficit in IGF-1R activation has been demonstrated in the muscle of old rats, associated with a reduced activity of the IRS1/PI3-K pathway (Haddad \& Adams 2006). Likewise, impaired AKT/PKB (protein kinase B) activity in myocytes has been found to be associated with higher levels of apoptosis, lower abundance of the contractile proteins myosin and actin, and a smaller muscle fibre crosssectional area (CSA) (Wu et al. 2009). The restoration of AKT kinase activity was associated with increased amount of contractile proteins and myocyte size, and decreased apoptotic rates. In addition, AKT-mediated phosphorylation of forkhead transcription factors (FOX) results in an inhibitory response of the transcription of several genes, among which is the atrogin-1 (also known as MAFbx) and MuRF1. These are two E3 ubiquitin ligases that promote muscle protein degradation mainly in presence of muscle inflammatory condition (Sandri et al. 2004). However, limited information from this area is available in humans.

An additional biological dysfunction of the elderly that contribute negatively to protein balance by increasing protein degradation is the chronic low-grade inflammation, a condition in which there is a two-to three-fold increase in the systemic concentration of inflammatory cytokines (Pedersen \& Febbraio, 2008). Infection and trauma generate acute inflammation, responding to an immune defence reaction, during which several cytokines are secreted to repair damaged tissues. However, prolonged inflammatory state, with elevated levels of cytokines such as IL-6, IL-1 $\beta$, CPR, IFN- $\gamma$ and TNF $\alpha$, has been proposed as a molecular process underlying the pathogenesis of many inactivity- and age-related diseases like metabolic syndrome, neurodegenerative diseases or muscle wasting. In addition, chronic inflammation is a contributing factor for agingrelated loss of physical function and onset of disability (Saini et al., 2009; Nicklas \& Brinkley, 2009). Inflammatory cytokines such as TNF $\alpha$ and IL-6, as well as reactive oxygen species (ROS), cortisol and angiotensin promote the ubiquitin-proteosome pathway, which is the most important mechanism for protein degradation in skeletal muscle cells (Lang et al., 2010). The elderly appear to have elevated expression of these inflammatory cytokines in skeletal muscle compared to younger subjects (Roubenoff, 2007).

On the other hand, oxidative metabolism generates ROS, a condition that can cause oxidative stress when there is an imbalance between oxidant and antioxidant molecular levels (Kregel \& Zhang, 2007). Oxidative stress seems to underlie the pathogenesis of chronic diseases and age-related skeletal muscle wasting, being involved in multifaceted pathways that stimulate protein breakdown (Bonetto et al., 2009). Mitochondrial dysfunction during aging increases the levels of ROS due to altered function of the respiratory chain and decreased cell defence against radicals (Short et al., 2005), what can cause direct damage to macromolecules such as lipids, proteins or nucleic acids. Mitochondrial dysfunction is related not only to loss of capacity to generate ATP but also to activation of pathways that lead to cellular apoptosis as in neurodegeneration or sarcopenia (Marzetti et al., 2008). Neurodegeneration during aging is another important contributor to the changes occurring on muscle structure (loss, atrophy and clustering of muscle fibres, as well as impaired sensory integration and motor response) due to denervation and loss of $\alpha$-motorneuron that result in a loss of motor units (Narici \& Maffulli, 2010).

In relation to the cellular defence from damage, Heat Shock Proteins (HSPs) are thought to be fundamental for the protection of cells, as well as for repair facilitation from injury and maintenance of cell homeostasis. They are present in low levels in unstressed skeletal muscle and increase in presence of stressors as oxidative stress, exercise, inflammation, and changes in $\mathrm{pH}$ or hyperthermia (Wallin et al., 2002). However, an attenuated HSPs response has been observed in several tissues, lymphocytes and monocytes from older rodents and humans following several stressors 
(Vasilaki et al., 2006; Njemini et al., 2003). This reduction appears to be related to changes in the pro-oxidant and antioxidant balance in the muscles of older individuals what may interfere in the activation of the adaptation-signalling response, therefore leading to functional deficits in the muscle fibre protection during ageing (Close et al., 2005).

\section{Physiological and Biological Benefits of Resistance Exercise for the Elderly}

Combating physical frailty in older age has become a major concern since the proportion of elderly citizens is exceeding that of young people. Maintaining mobility and independence until the end of chronological age through an active life-style should be the aspiration of every older individual. Although the biological aging process cannot be reversed by physical activity, it is likely that an individual who is physically active throughout the lifespan will have a greater reserve capacity to maintain high function into late life. There is evidence supporting that regular exercise can minimize the physiological effects of a sedentary lifestyle and can limit progression of some chronic diseases, loss of skeletal muscle mass and frailty (Kjaer \& Jespersen, 2009, Pedersen \& Saltin, 2006). In addition, the age-related physiological and functional changes become clinically and socially more relevant when disability impacts social roles and expectations.

Recent findings have shown that older adults who are less physically active are more likely to have lower skeletal muscle mass and strength with increased risk for sarcopenia (Rolland et al., 2008). Resistance training is the most important form of exercise intervention for people with sarcopenia. It can counteract the age-related changes in fibre type distribution and the decrease in fibre size (Klitgaard et al., 1990) by improving strength via neuromuscular adaptations and muscle hypertrophy in old and very old men and women (Little \& Phillips, 2009; Yarasheski et al., 1999).

Roth et al. (2001) demonstrated that 6 months of whole body resistance training in older people (65-75 years) produced gains in muscle cross-sectional area similar to those achieved in younger individuals aged 20-30 years. Kryger \& Andersen (2007) found that very old and frail men and women up to the age of 98 years respond to resistance training as younger and well-functioning elderly subjects do. They found significant increases in muscle strength, lean quadriceps CSA, type II fibre area and an overall increase in the relative amount of MHC IIA. Moreover, increases in the MHC protein synthesis after resistance training show that this type of exercise lead to increased protein synthesis and eventually to muscle hypertrophy in old and very old individuals, what is fundamental in their condition of muscle loss (Kryger \& Andersen, 2007). However, ageing is associated with lower myofibrillar protein synthesis in response to exercise and adequate amino acids levels compared to younger people, probably due to the mentioned blunted anabolic stimuli found in older people (Cuthberston et al., 2006).

In addition, a meta analysis of 121 randomized controlled trials of progressive resistance training in older people showed that training 23 times per week improved physical function, gait speed, timed get-up and go, climbing stairs and balance, and what was more important, muscle strength was especially improved in the high intensity training groups (Liu \& Latham, 2003). Resistance exercise appropriately executed increases muscle CSA as well as type 2 (fast twitch) muscle fibres. Increased type II fibre area in the major muscles of the legs is a very good clinical indicator in elderly subjects, since it may improve the rate of force development and power what is related with lower risk of suffering falls due to better postural control (Aagaard, 2003). As a result, this can lead to enhanced ability to perform activities of daily living, preventing functional decline and disability. Additionally, resistance exercise has been shown to improve different clinical conditions common in elderly people such as impaired glucose tolerance, elevated blood lipids, reduced resting metabolic rate and depression (Lang et al., 2010; Little and Phillips, 2009).

The benefits of life-long training for preventing age-related muscle loss are seen in master 
sprint-trained athletes who exhibit greater type I, IIA and IIB fibre areas compared to untrained controls (Korhonen et al., 2006). However, intense training (running or weight lifting) slows down but does not avoid the decline in muscle performance, the reduction in fast fibres size and the shift towards a slower MHC isoform profile that occur with aging (Korhonen et al., 2006; Faulkner et al., 2007). Regular resistance training prevents the loss of agerelated skeletal muscle mass by stimulating hypertrophy of the remaining fibres but it is not clear if loss in fibre number due to neuropathic processes or due to modification of the contractile proteins can be prevented (Narici \& Maffulli., 2010).

On the other hand, given the deleterious health effects of persistent inflammatory state, identification of therapies to its reduction appears critical, not only for controlling sarcopenia, but also for treating multiple adverse health conditions. Self-reported physical activity and aerobic fitness have been associated with reduced inflammatory cytokines (Colbert et al., 2004; Bruunsgaard, 2005; Beavers et al., 2010). A potential mechanism underlying the anti-inflammatory effect of regular physical activity is the production of myokines: cytokines produced by skeletal muscle (IL-6, IL-8, IL-15, BDNF, LIF) that create a systemic anti-inflammatory environment and work as hormones exerting endocrine effects on visceral fat and also within the muscle (Brandt \& Pedersen 2010). This fact suggests that muscle contraction has a central role in orchestrating metabolism and function of other organs while inactive lifestyle accompanies several body malfunctioning consequences.

There is recently further information from observational studies reporting that more frequent and more intense self-reported physical activity (Colbert et al., 2004; Elosua et al., 2005), or high aerobic fitness levels (Kullo et al., 2007; Arsenault et al., 2009) are associated with lower levels of certain inflammatory biomarkers in the elderly. On the other hand, in relation to data from intervention studies designed to examine the effects of training on inflammation, less consistent findings are found compared to observational studies. It is shown a trend toward lowering inflammation with exercise, but there is few data from fully powered, randomized, controlled trials to definitely conclude that regular exercise training reduce chronic inflammation in older adults (Nicklas \& Brinkley, 2009). More attention has been paid to the effects of aerobic exercise on the inflammatory risk profile and little information exists regarding resistance exercise (Phillips et al., 2009; Salles et al., 2010), with inconclusive results on the influence of resistance training on inflammatory marker levels in the elderly. It is possible that these results are dependent on study design characteristics, such as different intensity and duration of the exercise, absence of appropriate control group, combination of exercise types, low sample size, single cytokine measurements, etc. (Nicklas \& Brinkley, 2009).

In relation to the increased ROS production during exercise, initially considered responsible of induced muscle damage, it is now well recognised that physiological ROS production initiates muscle-endogenous adaptive responses able to protect the skeletal muscle cells against subsequent increases in ROS and damage. Muscle cells adapt to contraction and produce changes in the expression of cytoprotective responses by up-regulating the activity of antioxidant enzymes and HSPs, which may provide protection to further damaging events (Close et al., 2005). As molecular chaperones HSPs (HSP70, HSP27, $\alpha \mathrm{B}$-cristallin) are fundamental in facilitating the cellular remodelling processes subsequent to the training response (protein turnover, mitochondrial biogenesis, muscle hypertrophy, regulation of atrophic/apoptotic pathways) (Morton et al, 2009). HSPs expression is likely initiated after resistance or damaging exercise by the mechanical harm induced to the muscle proteins and is further augmented by the inflammatory response occurring during the recovery period.

In addition, persistent elevated oxidative damage up-regulates the antioxidant systems in elderly people. However, even if it may be operating at full capacity, it is not able to cope with the higher levels of oxidants produced in advanced age (Johnston et al. 2007). In this 
regard, the aged skeletal muscle is less prone to protect and regenerate injured tissue. This is related to the reduced anabolic signals regulated by IGF-1, but also to the altered redox balance observed in satellite cells that contributes to impair their myogenic properties (Bonetto et al., 2009).

Although the difficulties to cope with high levels of stress in the elderly, moderate exercise has been found to act as an antioxidant in young people by Gomez-Cabrera et al., (2008). They found that ROS generated during moderate exercise act as signal to increase the production of enzymes relevant for the adaptation of muscle cells to exercise. In addition, one investigation has demonstrated reduced levels of a lipid peroxidation marker (malondialdehyde) and an oxidant (hydroperoxide) after an acute aerobic exercise bout in resistance trained older individuals (Vincent et al., 2002), suggesting beneficial effects of resistance training on redox status in older people. In addition, low levels of serum/plasma antioxidant carotenoids are independently associated with low skeletal muscle strength and the development of walking disability (Samba et al., 2007). In relation to the cytoprotective proteins, following acute and chronic exercise HSPs may increase to recover cellular homeostasis and induce adaptation for further stress. Recent data indicate that chronic contractile activity induces increases in baseline HSP levels of cardiac and skeletal muscle of rodents (Morton et al., 2009).

We can conclude that encouraging the elderly to perform resistance training is beneficial for the individual quality of life in the later years what is also of great economical significance for the society, given the expanding costs going into care of the older individuals. The importance of exercise as a potential medicine against lifestyle associated diseases is growing since it has not side-effects for other systems as some drugs have (Kjaer \& Jespersen, 2009). Exercise causes adaptations to numerous systems at a metabolic, morphological and inflammatory level without negative effects; therefore it is never too late to adopt a healthy life-style through controlled exercise prescription, which seems to be a key to maintain body homeostasis. However, with respect to resistance training, more data is needed from human studies to verify the role of this intervention for the overall healthy status of elderly subjects.

\section{Future Perspectives}

Aging is a multifaceted process involving all levels of biological organization and may be a complex process to study, therefore it is interesting to elucidate weather the observed adaptation following resistance training are directly affecting the cellular mechanisms behind aging or simply covering the process. Does resistance training improve directly the health status or does it induce adaptations at the cellular and molecular levels that directly hamper the causes of aging?

Resistance training benefits have been related to improved physical function and independence in the elderly however more data is needed regarding its effects on several molecular processes. More randomized controlled studies are needed to refine the understanding of the effects of a resistance training protocol on systemic low-grade inflammation and on cytoprotective responses in elderly people. In the past, much research analyzed a reduced cluster of inflammatory cytokines related to certain disease condition; therefore there is a lack of research measuring multiple inflammatory biomarkers in humans, what would improve the health risk assessment. Moreover, it is not known the magnitude of this effect and the amount of training necessary to produce clinically significant effects for disease prevention. In relation to the effects on heat shock proteins, most of the data comes from studies analyzing aerobic training in human and animals but little is known about the stress response after progressive resistance training in humans. On the other hand, there are evidences that regular resistance training slows down the decline in muscle mass although more information is needed about the benefits to avoid loss of motor units.

This review summarizes recent and established knowledge about the age-related mechanisms affecting skeletal muscle decline. Resistance training is proposed as one of the most effective 
and healthy intervention benefiting the elderly individuals suffering muscle and physical degeneration. However, despite the extensive information in this area, we need to better understand the molecular mechanisms of atrophy and hypertrophy affected by the adaptations of resistance training. The concern of combating physical frailty in old age while maintaining an active lifestyle needs to be scientifically well supported.

\section{Acknowledgement}

This work was supported by University of Rome "Foro Italico", Research Grant 2009, and by Lazio Regional Municipality, to DC. RMBV is a $\mathrm{PhD}$ student in the European Doctoral Program in Health and Physical Activity.

\section{References}

Aagaard, P. (2003). Training-induced changes in neural function. Exercise and Sports Sciences Reviews, 131, 3161-317.

Arsenault, B.J., Cartier, A., Côté, M., Lemieux, I., Tremblay, A., Bouchard, C., Pérusse, L., Després, J.P. (2009). Body composition, cardiorespiratory fitness and low-grade inflammation in middle-aged men and women. American Journal of Cardiology, 104(2), 240246.

Bauer, J.M., Sieber, C.C. (2008). Sarcopenia and frailty: a clinician's controversial point of view. Experimental Gerontology, 43, 674-678.

Boire, Y. (2009). Physiopathological mechanism of sarcopenia. Journal of Nutrition Health and Aging, 13 (8), 717-723.

Bonetto, A., Penna, F., Muscaritoli, M., Minero, V.G., Fanelli, F.R., Baccino, F.M., Costelli, P. (2009). Are antioxidats useful for treating skeletal muscle atrophy? Free Radicals Biology and Medicine, 47 906-916.

Booth, F., Zwetsloot, K.A. (2009). Basic concepts about genes, inactivity and aging. Scandinavian Journal of Medicine and Science in Sports, 20, 1-4.
Brandt, C. \& Pedersen, B.K. (2010). The role of exercise-induced myokines in muscle homeostasis and the defence against chronic diseases. Journal of Biomedicine and Biotechnology, 2010, 520-52., doi:10.1155/2010/520258.

Burton, L.A., Sumukadas D. (2010). Optimal management of sarcopenia. Clinical interventions in aging, 5, 217-228.

Bruunsgaard, H. (2005). Physical activity and modulation of systemic low-level inflammation. Journal of Leukocyte Biology, 78, 819-835.

Carlson, M.E., Suetta, C., Conboy, M.J., Aagaard, P., Mackey, A., Kjaer, M., Conboy, I. (2009). Molecular aging and rejuvenation of human stem cells. EMBO molecular medicine, 1(8-9), 381-91.

Charge, S.B. \& Rudnicki, M.A. (2004). Cellular and molecular regulation of muscle regeneration. Physiological Reviews, 84 (1), 209-238.

Close, G.L., Kayani, A., Vasilaki, A., McArdle, A. (2005). Skeletal muscle damage with exercise and aging. Sports Medicine, 35(5), 413427.

Conboy, I.M., Conboy, M.J., Wagers, A.J., Girma, E.R., Weissman, I.L., Rando, T.A. (2005). Rejuvenation of aged progenitor cells by exposure to a young systemic environment. Nature, 433, 760-764.

Colbert, L.H., Marjolein, V., Simonsick, E., Tracy, R.P., Newman, A.B., Kritchevsky, S.B., Pahor, M., Taaffe, D.R., Brach, J., Rubin, S., Harris, T.B. (2004). Physical activity, exercise and inflammatory markers in older adults: findings from the health, aging and body composition study. Journal of the American Geriatrics Society, 52, 1098-1904.

Cuthberston, D.J., Babraj, J., Smith, K. (2006). Anabolic signalling and protein synthesis in human skeletal after dynamic shortening or lengthening exercise. American Journal of 
Physiology, Endocrinology and Metabolism, 290, E731-38.

Elosua, R., Bartali, B., Ordovas, J.M., Corsi, A.M., Lauretani, F., Ferrucci, L., InCHIANTI Investigators. (2005). Association between physical activity, physical performance, and inflammatory biomarkers in an elderly population: the InCHIANTI study. The Journals of Gerontology. Series A, Biological Sciences and Medical Sciences, 60(6), 760-767.

Faulkner, J.A., Larkin, L.M., Claflin, D.R. (2007). Age-related changes in the structure and function of skeletal muscles. Clinical and Experimental Pharmacology and Physiology, 34, 1091-1096.

Singh, M.A. (2004). Exercise and aging. Clinics in Geriatric Medicine, 20(2), 201-221.

Fried, L.P., Tangen, C.M., Walston, J., et al. (2001) Frailty in older adults: evidence for a phenotype. The Journal of Gerontoly. Series A, 56, M146-M156.

Gomez-Cabrera, M.C., Domenech, E., Viña, J. (2008). Moderate exercise is an antioxidant: upregulation of antioxidant genes by training? Free Radicals Biology and Medicine, 44, 126131.

Haddad, F. \& Adams, G.R. (2006). Agingsensitive cellular and molecular mechanisms associated with skeletal muscle hypertrophy. Journal of Applied Physiology, 100, 1188-1203.

Johnston, A.P.W., DeLisio, M., Parise, G. (2008). Resistance training, sarcopenia and the mitochondrial theory of aging. Applied Physiology, Nutrition and Metabolism, 33, 191 199.

Kjaer, M. \& Jespersen, J.G. (2009). The battle to keep it or to lose skeletal muscle with aging. The Journal of Physiology, 587, 1-2.

Klitgaard, H., Mantoni, M., Schiaffino, S., Ausoni, S., Gorza, L., Laurent-Winter, C., Schnohr, P., Saltin, B. (1990). Function, morphology and protein expression of ageing skeletal muscle: a cross-sectional study of elderly men with different training backgrounds. Acta Physiologica Scandinavica, 140, 41-54.

Korhonen, M.T., Cristea, A., Alen, M. et al. (2006). Aging, muscle fibre type and contractile function in sprint-trained athletes. Journal of Applied Physiology, 101, 906-917.

Kregel, K.C., Zhang, H.J. (2007). An integrated view of oxidative stress in aging: Basic mechanisms, functional effects, and pathological considerations. American Journal of Physiology- Regulatory, Integrative and Comparative Physiology, 292, R18-R36.

Kryger, A.I. \& Andersen, J.L. (2007). Resistance training in the oldest old: consequences for muscle strength, fibre types, fibre size, and MHC isoforms. Scandinavian Journal of Medicine and Science in Sports, 17, 422-430.

Kullo, I.J., Khalehi, M., Hensrud, D.D. (2007). Markers of inflammation are inversely associated with VO2 max in asymptomatic men. Journal of Applied Physiology, 102, 13741379.

Lang, T., Streeper, P., Cawthon, P., Baldwin, K., Taaffe, D.R., Harris, T.B. (2010). Sarcopenia: aetiology, clinical consequences, intervention and assessment. Osteoporosis International, 21, 543-559.

Little, J.P., Phillips, S.M. (2009). Resistance exercise and nutrition to counteract muscle wasting. Applied Physiology Nutrition and Metabolism, 34, 817-828.

Liu, C.J., Latham, N.K. (2003). Progressive resistance strength training for improving physical function in older adults. Cochrane Database System Review, CD002759.

Lord, S.R., McLean, D., Stathers, G. (1992) Physiological factors associated with injurious factors in older people living in the community. Gerontology, 38, 338-346. 
Luo, J., Sobkiw, C.L., Hirshman, M.F., Logsdon, M.N., Li, T.Q., Goodyear, L.J. \& Cantley, L.C. (2006). Loss of class IA PI3K signalling in muscle leads to impaired muscle growth, insulin response, and hyperlipidemia. Cell Metabolism, 3, 355-366.

Marzetti, E., Wohlgemutz, S.E., Lees, H.A., Chung, H., Giovannini, S., Leeuwenburgh, C. (2008). Age-related activation of mitochondrial caspase-independent apoptotic signalling in rat gastrocnemius muscle. Mechanisms of Ageing and Development, 129, 542-549.

Morton, J.P., Kayani, A.C., McArdle, A., Drust, B. (2009). The exercise-induced stress response of skeletal muscle, with emphasis on humans. Sports Medicine, 39(8), 643-662.

Narici, M.V., Maffulli, N. (2010). Sarcopenia, characteristics, mechanism, and functional significance. British Medical Bulletin, 95, 139159.

Nicklas, B.J. \& Brinkley, T.E. (2009). Exercise training as a treatment for chronic inflammation in the elderly. Exercise Sport Sciences Reviews, 27 (4), 165-170.

Njemini, R., Lambert, M., Demanet, C., Abeele, M.V., Vandebosch, S., Mets, T. (2003). The induction of HSP70 in peripheral mononuclear blood cells in elderly patients: a role for inflammatory markers. Human Immunology, 64(6), 575-585.

Pedersen, B.K. \& Saltin, B. (2006). Evidence for prescribing as therapy in chronic diseases. Scandinavian Journal of Medicine and Science in Sports, 16 (1), 3-63.

Pedersen, B.K. \& Febbraio, M.A. (2008). Muscle as an endocrine organ: focus on musclederived IL-6. Physiological Reviews, 88(4), 1379-1406.

Perrini, S., Laviola, L., Carreira, M.C., Cignarelli, A., Natalicchio, A., Giorgino, F. (2010). The GH/IGF1 axis and signalling pathways in the muscle and bone: mechanisms underlying age-related skeletal muscle wasting and osteoporosis. Journal of Endocrinology, 205 (3), 201-210.

Peterson, M.J., Giuliani, C., Morey, M.C. et al. (2009). Physical activity as a preventative factor for frailty: the health, aging, and body composition study. Journals of Gerontology. Series A, 64A(1), 61-68.

Phillips, S.M. (2007). Resistance exercise: good for more than just Grandma and Grandpa's muscles. Applied Physiology, Nutrition and Metabolism, 32, 1198-1205.

Phillips, M.D., Flynn, M.G., McFarlin, B.K., Stewart, L.K., Timmerman, K.L. (2009). Resistance training at eight-repetition maximum reduces the inflammatory milieu in elderly women. Medicine \& Sciences in Sports \& Exercise, 42(2), 314-325.

Puggaard, L. (2005). Age-related decline in maximal oxygen capacity: consequences for performance of everyday activities. Journal of the American Geriatrics Society, 53 (3), 546547.

Robertson, K., Kopchick, J.J. \& Liu, J. (2006). Growth hormone receptor gene deficiency causes delayed insulin responsiveness in skeletal muscles without affecting compensatory islet cell overgrowth in obese mice. American Journal of Physiology, Endocrinology and Metabolism,29, E491-E498.

Rolland, Y., Czerwinski, S., Van Kan, G.A., et al. (2008). Sarcopenia: its assessment, aetiology, pathogenesis, consequences and future perspectives. The Journal of Nutrition, Health and Aging, 12, 433-450.

Rosenberg, I.H. (1997). Sarcopenia: origins and clinical relevance. American Society for nutritional Sciences, 127, 9905-9915.

Roth, S.M., Martel, G.F., Ivey, F.M. (2001). Skeletal muscle satellite cell characteristics in young and older men and women after heavy resistance strength training. The Journals of Gerontology. Series A, Biological Sciences and Medical Sciences, 56, B240-B247. 
Roubenoff, R. (2007). Physical activity, inflammation and muscle loss. Nutrition Reviews, 65, S208-S212.

Saini, A., Faulkner, S., Al-Shanti, N., Stewart, C. (2009). Powerful signals for weak muscles. Ageing Research Reviews, 8(4), 251-267.

Salles, B.F., Simao, R., Fleck, S.J., KraemerAguiar, L.G., Bouskela, E. (2010). Effects of resistance training on cytokines. International Journal of Sports Medicine, 31(7), 441-450.

Samba, R.D., Lauretani, F., Ferrucci, L. (2007). Carotenoids as protection against sarcopenia in older adults. Archives of Biochemistry and Biophysics, 458, 141-145.

Sandri, M., Sandri, C., Gilbert, A., Skurk, C., Calabria, E., Picard, A., Walsh, K., Schiaffino, S., Lecker, S.H. \& Goldberg, A.L. (2004). Foxo transcription factors induce the atrophy-related ubiquitin ligase atrogin-1 and cause skeletal muscle atrophy. Cell, 117, 399-412.

Short, K.R., Bigelow, M.L., Kahal. J. (2005). Decline in skeletal muscle mitochondrial function with aging in humans. Proceedings of the National Academy of Sciences of USA, 102, 5618-2.

Sturnieks, D.L., George, R.S., Lord, S.R. (2008). Balance disorders in the elderly. Clinical Neurophysiology, 38, 467-478.

Vincent, K.R., Vincent, H.K., Braith, R.W., Lennon, S.L. \& Lowenthal, D.T. (2002). Resistance exercise training attenuates exercise- induced lipid peroxidation in the elderly. European Journal of Applied Physiology, 87, 416-423.

Vasilaki, A., McArdle, F., Iwanejko, L.M., McArdle, A. (2006). Adaptive responses of mouse skeletal muscle to contractile activity: the effect of age. Mechanisms of Aging Development, 127(11), 830-839.

Wallin, R.P., Lundqvist, A., More, S.H., von Bonin, A., Kiessling, R., Ljunggren, H.G. (2002). Heat-shock proteins as activators of the innate immune system. Trends in Immunology, 23, 130-135.

WHO, International Classification of Functioning, Disability and Health, 2001. www.who.int/classifications/icf/

accessed17.12.2007

Wu, M., Katta, A., Gadde, M.K., Liu, H., Kakarla, S.K., Fannin, J., Paturi, S., Arvapalli, R.K., Rice, K.M., Wang, Y., et al. (2009). Aging-associated dysfunction of Akt/protein kinase B: S-nitrosylation and acetaminophen intervention. PLoS ONE, 2009; 4: e6430.

Yarasheski, K.E., Pak-loduca, J., Hasten, D.L., Obert, K.A., Brown, M.B., Sinacore, D.R. (1999). Resistance exercise training increases mixed muscle protein synthesis rate in frail women and men more than $76 \mathrm{yr}$ old. American Journal of Physiology, Endocrinology and Metabolism, 277,118-125. 